\title{
DIMENSIÓN PSICOSOCIAL DE LA PANDEMIA: LA OTRA CARA DEL COVID-19
}

\author{
PSYCHOSOCIAL DIMENSION OF THE PANDEMIC: THE OTHER SIDE \\ OF COVID-19
}

\section{DIMENSÃO PSICOSSOCIAL DA PANDÊMICA: O OUTRO LADO DE COVID-19}

\author{
Ana María LeIva* \\ Gabriela NAZAR** \\ María Adela MartíneZ-Sanguinetti**** \\ FAnny Petermann-Rocha**** \\ JoRgELINA RicCHEZZA***** \\ Carlos Celis-Morales S***** $^{* * *}$
}

\begin{abstract}
RESUMEN
Objetivos: Identificar las consecuencias psicosociales de la pandemia y definir propuestas para sobrellevar los efectos negativos de la pandemia y el confinamiento en la salud mental. Material y Método: Para el primer objetivo se realizó una búsqueda de artículos empíricos en inglés o espańol, indexados en las bases de datos Web of Science, publicados entre enero y mayo de 2020. Para el segundo objetivo se amplió la búsqueda, desde el año 2004 en adelante, a revisiones, encuestas nacionales, comunicaciones breves y reportes de organismos internacionales, tales como la OMS, el Centro de Control de Enfermedades de los Estados Unidos (CDC), y el Centro Europeo de Control de Enfermedades (ECDC). Resultados: Se analizaron 11 artículos científicos y 6 documentos. La experiencia emocional ante la pandemia es de amplia variabilidad individual, reportándose alta prevalencia de angustia psicológica y ansiedad. La severidad de los síntomas se relacionó
\end{abstract}

*Bióloga. Mg. en Neurociencias y Salud Mental. Instituto de Anatomía, Histología y Patología, Facultad de Medicina, Universidad Austral de Chile, Valdivia, Chile. ORCID: https://orcid.org/0000-0001-8390-6366. Email: aleiva@uach.cl. Autor correspondencia.

**Psicóloga, PhD. Departamento de Psicología y Centro de Vida Saludable, Universidad de Concepción. Concepción, Chile. ORCID: https://orcid.org/0000-0002-6410-6838. Email: gnazar@udec.cl. Autor Correspondencia

***Bioquímica. MSc. Nutrición y Dietética. Instituto de Farmacia, Facultad de Ciencias, Universidad Austral de Chile, Valdivia, Chile. ORCID: https://orcid.org/0000-0002-7609-7705. Email: mmartin3@uach.cl

${ }^{* * * *}$ Nutricionista. MSc. Nutrición Humana. Institute of Health and Wellbeing, BHF Glasgow Cardiovascular Research Centre, Institute of Cardiovascular and Medical Sciences, University of Glasgow, Glasgow, United Kingdom. ORCID: https://orcid. org/0000-0002-4384-4962. Email: 2348299P@student.gla.ac.uk

*****Licenciada en Psicología. Instituto de Estudios Gerontológicos, Universidad Atlántida Argentina. Buenos Aires, Argentina. ORCID: https://orcid.org/0000-0001-7156-2762. Email: jorgelina_ric@hotmail.com

****** Profesor de Educación Física, Dr. en Ciencias Cardiovasculares y Biomédicas. BHF Glasgow Cardiovascular Research Centre, Institute of Cardiovascular and Medical Sciences. University of Glasgow. Glasgow, United Kingdom. ORCID: https:// orcid.org/0000-0003-2612-3917. Email: Carlos.Celis@glasgow.ac.uk 
con la duración del aislamiento, miedo a contraer la enfermedad, inestabilidad financiera y exposición a los medios de comunicación. Para el manejo de las consecuencias adversas, se propone cautelar la satisfacción de necesidades básicas, mantener vínculos sociales y recibir información veraz y no alarmista de parte de las autoridades y medios de comunicación. Conclusión: Las consecuencias de la pandemia incluyen malestar y respuestas de temor y ansiedad. En las personas en las que se conjugan situaciones de abandono y exclusión social, el confinamiento precariza aún más esta condición. Considerando que la pandemia se da en un contexto de alta heterogeneidad, la dimensión psicosocial debe constituir un eje de acción prioritario.

Palabras clave: Aislamiento social; COVID-19; Pandemia; Salud mental.

\begin{abstract}
Objective: To identify the psychosocial consequences of the pandemic and to define proposals for coping with the negative effects of the pandemic and confinement on mental health. Materials and Methods: For the first objective we searched for empirical articles in English or Spanish, published between January and May 2020, and indexed in the Web of Science data base. For the second objective, the search was expanded, from the year 2004 onwards, to reviews, national surveys, short communications and reports from international organizations such as the WHO, the United States Center for Disease Control (CDC), and the European Centre for Disease Control (ECDC). Results: 11 scientific articles and 6 documents were analyzed. The emotional experience related to the pandemic has a wide range of individual variability, with a high prevalence of psychological distress and anxiety reported. The severity of symptoms was related to the period of isolation, fear of contracting the disease, financial instability and media exposure. In order to manage adverse consequences, it is recommended to satisfy basic needs, maintain social ties and receive truthful and non-alarmist information from the authorities and the media. Conclusion: The consequences of the pandemic include discomfort and responses of fear and anxiety. For people who suffer abandonment and social exclusion, confinement makes this condition even more precarious. Considering that the pandemic occurs in a context of high heterogeneity, the psychosocial dimension should also be a priority.
\end{abstract}

Key words: Social isolation; COVID-19; Pandemic; Mental health.

\title{
RESUMO
}

Objetivo: Identificar as consequências psicossociais da pandemia e definir propostas para lidar com os efeitos negativos da pandemia e o confinamento na saúde mental. Material e método: Para o primeiro objetivo foi realizada uma busca de artigos empíricos em inglês ou espanhol, indexados no banco de dado Web of Science, publicados entre janeiro e maio de 2020. Para o segundo objetivo a busca foi ampliada, da partir de 2004, a revisôes, pesquisas nacionais, breves comunicaçóes e relatórios de organizaçóes internacionais, tais como OMS, Centro de Controle e Prevenção de Doenças dos Estados Unidos (CDC), e o Centro Europeu de Controle de Doenças (ECDC). Resultados: 11 artigos científicos e 6 documentos foram analisados. A experiência emocional ante a pandemia é de grande variabilidade individual, relatando uma alta prevalência de sofrimento psíquico e ansiedade. A gravidade dos sintomas estava relacionada à duração do isolamento, medo de contrair a doença, instabilidade financeira e exposiçáo à mídia. Para gerenciar as consequências adversas, propóe-se proteger a satisfação de necessidades básicas, manter laços sociais e receber informação verdadeira e não alarmista das autoridades e da mídia. Conclusáo: As consequências da pandemia incluem desconforto e respostas de medo e ansiedade. Nas pessoas em situação de abandono e exclusão social, o confinamento torna essa condição ainda mais precária. Considerando que a pandemia ocorre em um contexto de alta heterogeneidade, a dimensão psicossocial deve constituir um eixo prioritário de açáo.

Palavras-chave: Isolamento social; COVID-19; Pandemia; Saúde mental.

Fecha de recepción: 26/05/2020

Fecha de aceptación: 18/07/20 


\section{INTRODUCCIÓN}

Cuando se informó del brote de la enfermedad de coronavirus (COVID-19, del inglés Coronavirus Diseases), en la provincia de Hubei (Wuhan, China) a fines del año 2019, nadie podía predecir que estábamos ad portas de una de las mayores crisis sanitarias y humanitarias de nuestros tiempos.

El aumento exponencial de casos registrados y de países afectados por el virus SARS-CoV-2 (del inglés Severe Acute Respiratory Syndrome Coronavirus 2), que causa la enfermedad COVID-19, llevó a que el 30 de enero de 2020, la Organización Mundial de la Salud (OMS) declarase una Emergencia de Salud Pública de Importancia Internacional ${ }^{(1)}$.

Las consecuencias globales alcanzadas por la rápida expansión derivó a que el 20 de marzo de 2020, el COVID-19, pasara a la categoría de pandemia. Decisión que si bien fue tardía, presenta absoluta validez considerando el actual escenario a nivel mundial. A la fecha (25 de mayo de 2020) se registran 5.460.747 casos confirmados y 346.293 muertes a causa del COVID-19 a nivel mundial ${ }^{(2)}$.

A pesar de que el origen exacto del COVID-2019 aún está en discusión, se sabe que es un virus con un alto nivel de propagación entre seres humanos, favorecido principalmente por la proximidad entre personas, lo que denotó en que en vastas regiones del mundo se decretaran cuarentenas preventivas y el consecuente aislamiento social. Todo esto como una medida para disminuir la propagación del virus a través de la interacción humana y preparar a los sistemas de salud para una mejor contención de la pandemia ${ }^{(3)}$.

La elevada cantidad de contagiados y muertes, sumado a las importantes pérdidas económicas que se dan en el contexto de una pandemia, generan un significativo riesgo psicosocial, especialmente en poblaciones que poseen escasos recursos, viven en condiciones precarias y tienen limitado acceso a los servicios sociales y de salud ${ }^{(4)}$.

En la medida que las personas se aíslan, las estructuras de apoyo social se rompen, perdiendo el intercambio con otros elementos significativos de nuestro entorno como son los familiares, amigos, organizaciones comunitarias o laborales, que actúan, habitualmente, como fuente de soporte emocional y material ${ }^{(5)}$.

Si bien el COVID-19 se propaga sin discriminación entre los seres humanos, hay grupos más vulnerables, entre ellos, las personas mayores, quienes presentan un mayor riesgo de desarrollar sintomatología severa debido a sus comorbilidades. En este sentido, la $\mathrm{OMS}^{(6)}$ ha insistido en la necesidad de garantizar que la población adulta mayor sea protegida, mediante el aislamiento o restricción de interacción social; recomendación que no debe ser confundida con la segregación que ponga en riesgo sus derechos fundamentales.

A la fecha, la información asociada a la emergencia se ha centrado fundamentalmente en aspectos epidemiológicos y clínicos, existiendo un vacío de conocimiento respecto de las consecuencias psicosociales que la pandemia está generando. Contar con estudios que generen información, es una necesidad que permitirá identificar los problemas más inmediatos y desarrollar estrategias de mitigación, especialmente en población más vulnerable. Según lo anterior, el objetivo de esta revisión fue identificar las consecuencias psicosociales de la pandemia por COVID-19, reportadas en la literatura reciente, y definir propuestas que contribuyan a sobrellevar los efectos negativos de la pandemia y el confinamiento en la salud mental.

\section{MATERIAL Y MÉTODOS}

Revisión narrativa de la literatura a partir de 17 documentos: artículos originales, reportes, revisiones de literatura, encuestas de representatividad nacional, realizadas en otros países afectados por COVID-19, y reportes de organizaciones internacionales como la OMS, el Centro de Control de Enfermedades de Estados Unidos (CDC), y el Centro Europeo de Control de Enfermedades (ECDC).

Para informar los resultados de las consecuencias psicosociales de la pandemia se revisó la base de datos: Web of Science, utilizando operadores booleanos en la siguiente estrategia de búsqueda: Coronavirus OR quarentine AND psychological effects OR psychosocial OR distress OR mental health OR mental health care, y sus equivalentes en idioma español, en título y abstract, en artículos científicos publicados entre el 1 de enero y 15 de mayo del año 2020. Como criterios de exclusión se consideró: a) estudios en grupos distintos a población general, ejemplo: población clínica, 
estudiantes, personal de salud. Se identificaron 11 estudios $^{(7-17)}$ (Figura 1).

Para las propuestas en el manejo de la pandemia, se desarrolló una segunda búsqueda que incluyó revisiones, comunicaciones breves, encuestas y reportes de organizaciones internacionales desde el año 2004 en adelante, los que fueron seleccionados en términos de su pertinencia con el objetivo de esta revisión. Se identificó un total de 6 documentos.

El proceso de búsqueda, identificación y verificación del cumplimiento de criterios de los estudios fue realizado de manera independiente por dos integrantes del equipo de investigación, selección que posteriormente fue ratificada por un tercer evaluador.

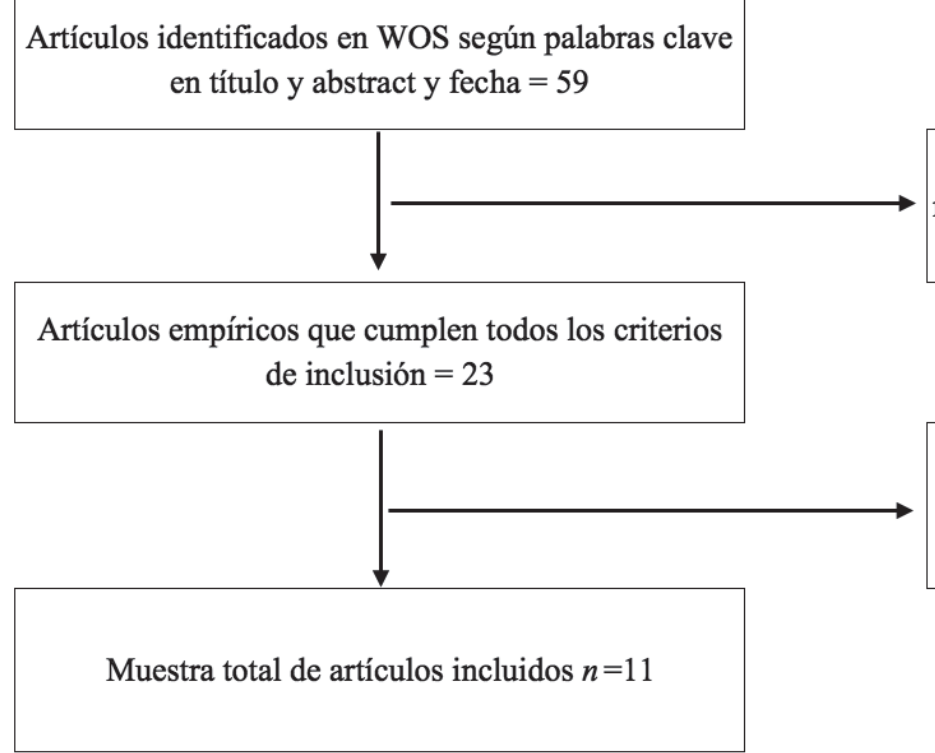

Excluidos artículos no empíricos: revisiones, cartas al editor, puntos de vista $\mathrm{y}$ comentarios $=36$ población general: Personal sanitario $=9$ Estudiantes $=1$

Figura 1. Flujograma del proceso de selección de artículos científicos.

\section{RESULTADOS}

Consecuencias en salud mental asociadas al COVID-19. Se analizaron 11 artículos originales de tipo transversal que investigaron las consecuencias psicosociales de la pandemia por COVID-19, en población general, la mayor parte de ellos en Asia (Tabla 1) ${ }^{(7-17)}$.

Si bien aún es muy pronto para dimensionar las consecuencias psicosociales que dejará la pandemia a largo plazo, estudios empíricos incipientes y reportes poblacionales están dando cuenta de sus afectos en diversos aspectos de la vida de las personas, que han desencadenado una amplia variedad de problemas psicológicos, entre ellos presencia de sintomatología ansiosa y depresiva ${ }^{(7-17)}$. Dichos efectos tienen relación directa con las consecuencias prácticas de las medidas implementadas para controlar la pandemia, como modificaciones y restricciones en las actividades del diario vivir, en el ámbito de transporte, contacto social, trabajo, entre otros; sumado a las implicaciones económicas ${ }^{(7-17)}$.

Respecto de la sintomatología depresiva, la investigación de Zhang y cols. en 205 residentes de la ciudad de Zhongshan, China, informó que, tanto los pacientes que fueron afectados por COVID-19, como el público en general, sin infección, presentaron síntomas depresivos severos, con un 19,3 y $14,3 \%$ en cada grupo, respectivamente ${ }^{(12)}$. Otro estudio liderado por Wang y cols. ${ }^{(8)}$, informó que un $16,5 \%$ de la población encuestada (1.219 participantes) presentaba sintomatología depresiva moderada a severa. Estos valores son similares a los informados por Wang y cols., en otro estudio, también en población China, que encuestó a 600 participantes, quienes reportaron un $17,2 \%$ de sintomatología depresiva ${ }^{(15)}$. 


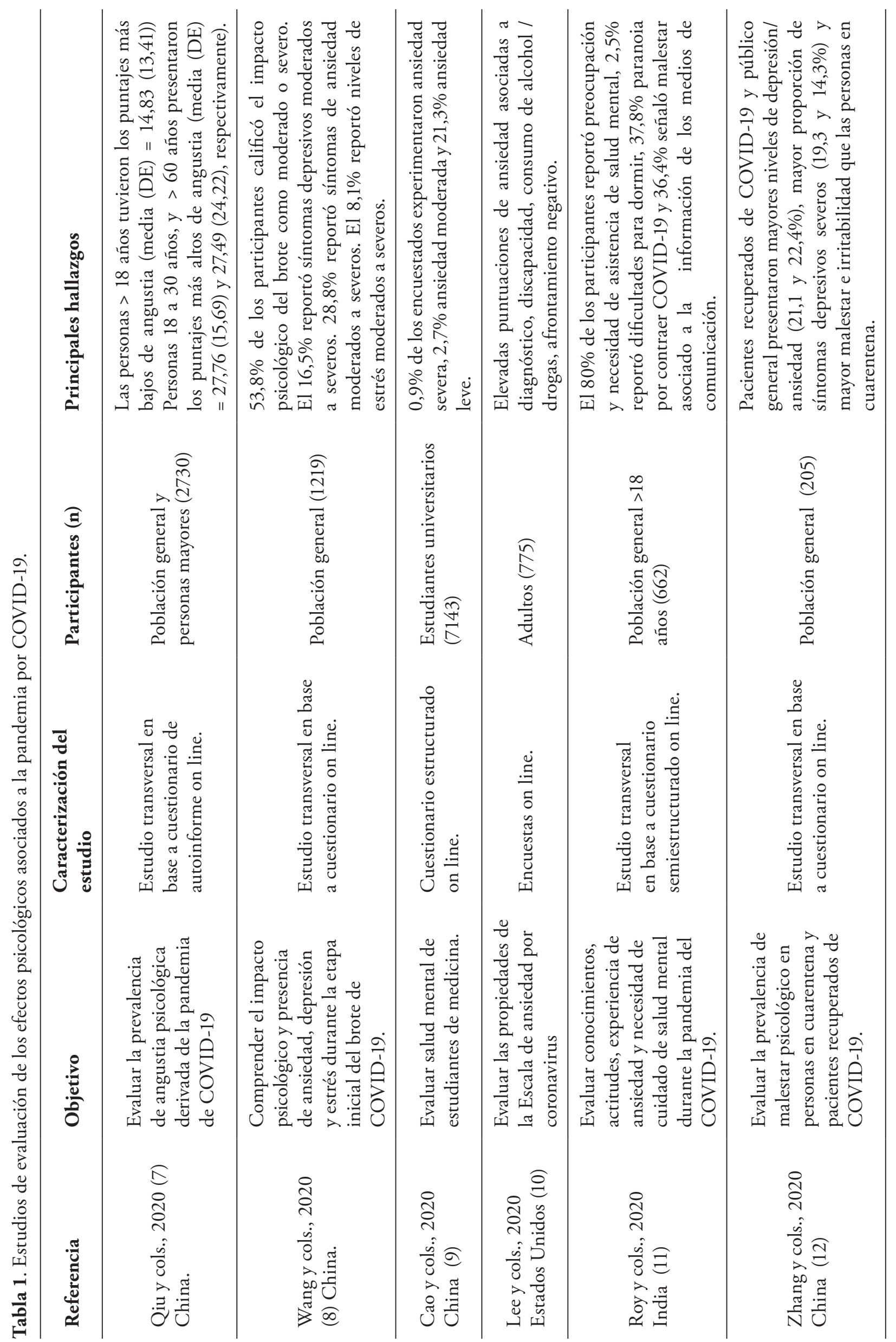




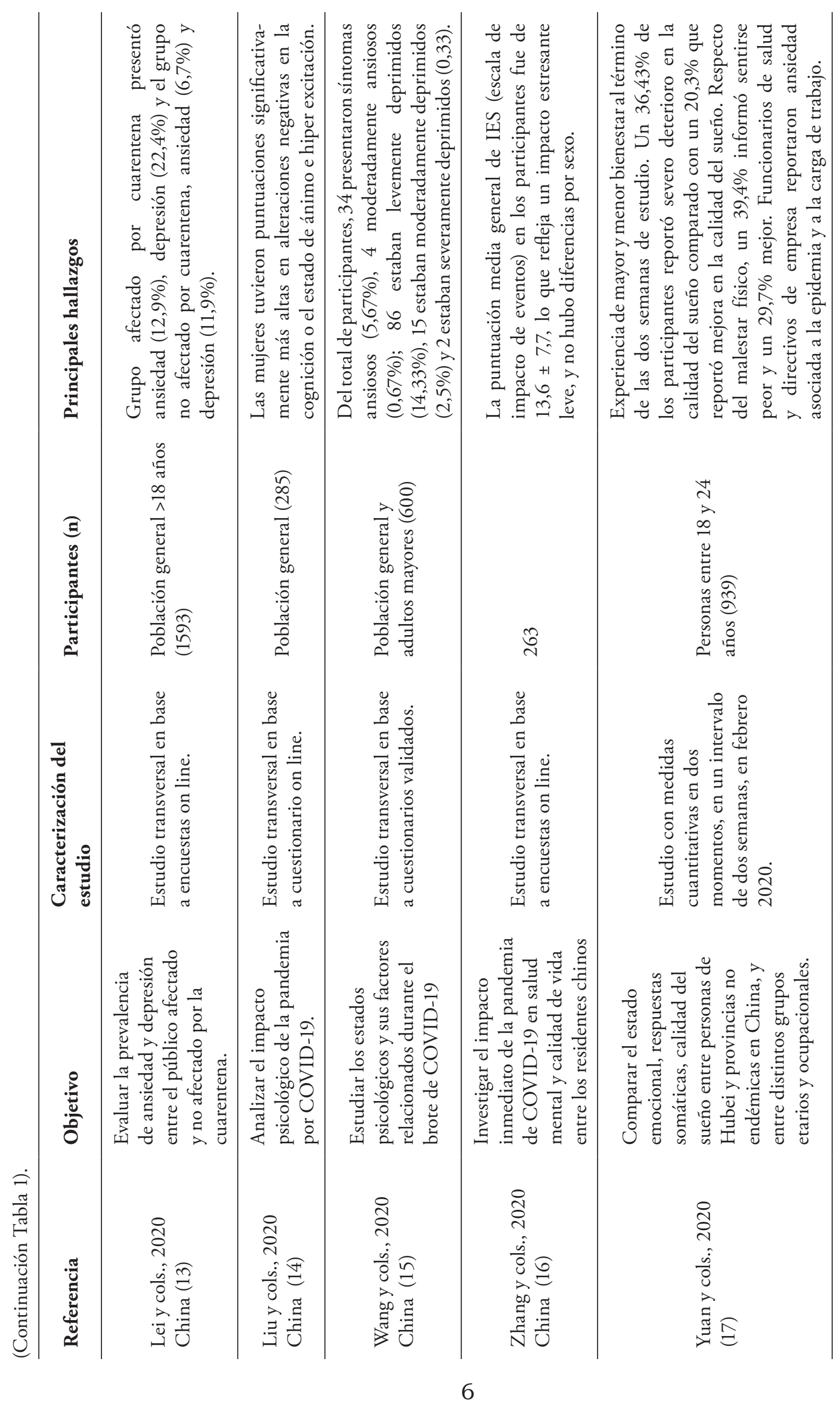


Al comparar los resultados de los diferentes estudios revisados, existe coincidencia en relación con la presencia de síntomas de angustia psicológica y ansiedad. Dicha sintomatología se asoció a factores como género, falta de apoyo social, escasos recursos de afrontamiento, miedo a infectarse, duración del aislamiento, inseguridad económica y cantidad de exposición a los medios de comunicación ${ }^{(9,10,12,13)}$.
Orientaciones propuestas en la literatura para el manejo de los efectos psicosociales de la pandemia. Se analizaron 9 documentos a partir de los cuales se extrajeron orientaciones para el manejo de las consecuencias psicológicas de la pandemia, las que se resumen en la Tabla $2^{(18-23)}$.

Tabla 2. Resumen de propuestas para el manejo de los efectos psicosociales de la pandemia COVID-19, a niveles gubernamental, social-comunitario e individual.

\begin{tabular}{|c|c|}
\hline \multirow{8}{*}{$\begin{array}{l}\text { A nivel } \\
\text { gubernamental }\end{array}$} & $\begin{array}{l}\text { Establecer medidas de cuarentena o confinamiento total de corta duración y enfatizar su } \\
\text { carácter altruista (18) }\end{array}$ \\
\hline & $\begin{array}{l}\text { Cautelar la intersectorialidad en el diseño e implementación de medidas de mitigación de } \\
\text { los efectos de la pandemia en la salud mental }(19,20,21)\end{array}$ \\
\hline & $\begin{array}{l}\text { Entregar información clara y oportuna, enfatizando las razones a la base de las medidas } \\
\text { implementadas }(18,19,22)\end{array}$ \\
\hline & $\begin{array}{l}\text { Asegurar provisión de alimentos, higiene, seguridad y prestaciones básicas de salud para la } \\
\text { población }(18,23)\end{array}$ \\
\hline & Disponer de material psicoeducativo simple y accesible para la población (19) \\
\hline & Favorecer el acceso a tecnologías de información y conectividad $(18,19)$ \\
\hline & $\begin{array}{l}\text { Asegurar el acceso a salud mental especializada a grupos vulnerables como personas mayores, } \\
\text { personas con antecedentes de enfermedades mentales, personas recuperadas de la infección } \\
\text { y familiares de personas fallecidas (19) }\end{array}$ \\
\hline & $\begin{array}{l}\text { Implementar estrategias de promoción y prevención de problemas de salud mental en los } \\
\text { equipos de salud (20) }\end{array}$ \\
\hline \multirow{5}{*}{$\begin{array}{l}\text { A nivel } \\
\text { social-comunitario }\end{array}$} & $\begin{array}{l}\text { Mantener activas las redes territoriales, comunitarias y organizaciones sociales existentes en } \\
\text { la comunidad ( } 21)\end{array}$ \\
\hline & $\begin{array}{l}\text { Fortalecer las capacidades de las organizaciones sociales para educar a la comunidad de } \\
\text { formas que sean social y culturalmente pertinentes (21) }\end{array}$ \\
\hline & $\begin{array}{l}\text { Implementar medidas de promoción de la salud mental y prevención de las consecuencias } \\
\text { psicosociales de la pandemia en las comunidades escolares (21) }\end{array}$ \\
\hline & $\begin{array}{l}\text { Establecer redes de colaboración y asistencia a grupos vulnerables (inmigrantes, personas } \\
\text { mayores, cuidadores de personas dependientes, entre otros) }(19,20,21)\end{array}$ \\
\hline & Censurar la estigmatización de personas infectadas o grupos raciales (18) \\
\hline \multirow{6}{*}{ A nivel individual } & $\begin{array}{l}\text { Comprender que el malestar psicológico, miedo y tristeza son respuestas normales frente a } \\
\text { una situación desconocida }(18,19)\end{array}$ \\
\hline & $\begin{array}{l}\text { Informarse a través de medios oficiales y no difundir información de fuentes no confiables } \\
(18,19,20,22)\end{array}$ \\
\hline & Mantener cuidado de la salud y uso de prescripciones médicas habituales (19) \\
\hline & $\begin{array}{l}\text { Estar atento y pedir ayuda cuando se presentan síntomas persistentes y agudos de ansiedad, } \\
\text { tristeza o irritabilidad; conductas innecesarias y compulsivas de higiene o monitoreo de la } \\
\text { salud, y abuso de alcohol o drogas (19) }\end{array}$ \\
\hline & $\begin{array}{l}\text { Mantener hábitos y rutinas de cuidado personal, sueño, actividad física y actividades de ocio } \\
\text { y esparcimiento (19) }\end{array}$ \\
\hline & $\begin{array}{l}\text { Desarrollar actividad física y la meditación por sus comprobados efectos beneficiosos en el } \\
\text { bienestar (19) }\end{array}$ \\
\hline
\end{tabular}


Uno de los aspectos más importantes a cautelar es que la satisfacción de las necesidades fundamentales no se vea severamente interferida, especialmente en población más vulnerable. Consecuentemente, proveer ayuda instrumental, particularmente con la provisión de alimentos, insumos de higiene, atención de salud y medicamentos, es una condición básica y necesaria ${ }^{(18,19)}$.

El contacto social y la percepción de apoyo social están dentro de los factores protectores de la salud más efectivos, cuyo efecto cobra mayor relevancia en situaciones de catástrofe o elevado estrés. Consecuentemente, mantener los vínculos en la modalidad que sea posible, aun cuando no sea cara a cara, otorga a las personas un sentido de protección que contribuye a su bienestar ${ }^{(8,18,19,24)}$.

La resiliencia, definida como la capacidad de adaptación frente a un agente perturbador o estado o situación adversa, es otro aspecto de la personalidad considerado un factor protector, al igual que la espiritualidad que puede ser una fuente importante de alivio y contención para muchas personas, y una forma de dar sentido a la situación actual $^{(18,25)}$.

La ubicación del sujeto, como un eslabón en la cadena generacional, es un factor protector que en el caso de las personas mayores puede contribuir a que no se sientan excluidas. Estas personas han sorteado situaciones difíciles en su vida, pudiendo aportar con su experiencia a través de la generatividad; de ahí que hay que procurar mantener un contacto, a la distancia, pero afectivo, que les permita seguir socializando con otros significativos ${ }^{(18-21,25)}$.

Se destaca además la importancia de que los funcionarios de salud, y sus referentes, enfaticen en la población lo altruista de la elección del autoconfinamiento, lo que contribuye a dar tranquilidad, al sentir que están aportando ${ }^{(18,20,21)}$.

Finalmente, la entrega de información confiable, consistente y no alarmista, particularmente respecto al tiempo de confinamiento y las medidas de resguardo, ayudan a la sensación de control. Consecuentemente, la información que se entrega a la población debe ser coherente, organizada y de común acuerdo, principalmente por parte de entidades gubernamentales, personajes públicos, y principalmente los medios de comunicación ${ }^{(18,19}$, 20,26).

\section{DISCUSIÓN}

Si bien aún no se conoce la real dimensión de la pandemia por COVID-19, y sus consecuencias psicosociales, se identificó un amplio conjunto de revisiones e informes de expertos, que vinculan el aislamiento social y la soledad con una mala salud mental ${ }^{(18,19,21,22)}$.

Resultados preliminares basados en estudios poblacionales realizados en países inicialmente afectados por COVID-19, revelan que las consecuencias psicosociales de la pandemia en la población son variables, sin embargo, existe coincidencia en la presencia de estrés, angustia psicológica, ansiedad, depresión y otras manifestaciones de malestar ${ }^{(7-17,27)}$.

Proyecciones de la OMS estiman que entre un $3-4 \%$ de la población mundial sufrirá trastornos psicológicos severos y entre un 15-20\% podría padecer trastornos leves a moderados. Entre tanto, el porcentaje restante se verá expuesto a un fuerte estrés psicosocial no patológico, cuyos efectos trascenderán la pandemia ${ }^{(6)}$.

Esta información es ratificada por resultados incipientes de investigaciones que se están generando a partir de encuestas online, que evidencian que la pandemia afecta negativamente la salud mental de la población y aumenta el malestar psicológico ${ }^{(7-17)}$. Esto es preocupante, ya que se ha reportado que el aislamiento social y la soledad se asocian con un mayor riesgo de desarrollar enfermedad coronaria y accidente cerebrovascular, independientemente de los factores de riesgo tradicionales de enfermedad cardiovascular ${ }^{(28)}$.

Datos generados en el Reino Unido durante la pandemia indican que, si bien el aumento en síntomas de ansiedad y estrés son respuestas esperadas en la población durante periodos de confinamiento $^{(18)}$, las personas con antecedentes clínicos de ansiedad o depresión presentarían mayor probabilidad de experimentar conductas autodestructivas autolesivas o suicidio ${ }^{(29)}$. Este tipo de conductas ya se han reportado en emergencias sanitarias anteriores ${ }^{(30)}$ como fue la gripe del 2003 ocurrida en Hong-Kong, asociada al virus SARS (del inglés Severe Acute Respiratory Syndrome), donde se documentó un $30 \%$ de incremento de los casos de suicidio, especialmente en personas mayores de 65 años. Se constató además que 
un $50 \%$ de los pacientes recuperados del SARS permanecieron con cuadros de ansiedad posterior a su recuperación ${ }^{(30,31)}$. Por su parte, las personas que estuvieron en riesgo vital presentaron un mayor riesgo de estrés postraumático y depresión y un $29 \%$ de los trabajadores de recintos hospitalarios presentaron estrés emocional ${ }^{(31,32)}$.

Otro impacto en la salud mental de la población es la inseguridad económica, debido al incremento del desempleo e imposibilidad de la población más desprotegida socialmente, de desarrollar actividades de trabajo informal, producto del confinamiento, lo cual contribuye a incrementar los niveles de ansiedad y estrés ${ }^{(22,33)}$.

Si bien la pandemia del COVID-19 tiene consecuencias en la salud mental en población general, hay evidencia que indica que los grupos más vulnerables (niños, personas mayores, personas con comorbilidades, con discapacidad y aquellas con antecedentes de problemas de salud mental) presentan consecuencias más severas ${ }^{(22)}$.

En el ámbito social, un estudio poblacional en Latinoamérica, realizado en Argentina, recabó información en diferentes provincias, respecto al impacto del COVID-19 $9^{(23)}$. Dicho informe da cuenta de las dificultades que experimenta la población para cumplir con las medidas de aislamiento, como las carencias estructurales sufridas por vastos sectores del país, en cuanto a la elevada marginalidad, precariedad ocupacional y desempleo. Se reporta además agravamiento de la situación de aislamiento de la población mayor en contextos socioeconómicos diversos, la que se ha visto intensificada por sus limitaciones para acceder a las redes sociales ${ }^{(23)}$.

Otro aspecto fundamental para destacar es el reto que implica para los diferentes países y regiones enfrentar esta pandemia en un contexto de disparidades sociales, lo que se ha hecho más evidente en estos días de confinamiento. La marginalidad, condiciones de hacinamiento, y falta de servicios básicos, están siendo un verdadero reto para muchos de los gobiernos ${ }^{(23)}$. Esta realidad contrasta con quienes, a pesar del aislamiento social, tienen la tranquilidad que les confiere un respaldo económico, y un hogar con las comodidades y recursos que les permiten sobrellevar el encierro.

En base a estos antecedentes, la pandemia no impactará de la misma forma a una persona que tenga garantizada la protección de sus derechos y determinantes sociales (vivienda, alimentación, ingresos, saneamiento, afectos, redes sociales, estado de salud, entre otros), en comparación con otras que no los tengan (personas en situación de calle, personas mayores que viven solas, con ingresos precarios, deterioro cognitivo o aislamiento afectivo) ${ }^{(34)}$.

Consecuentemente, la emergencia sanitaria actual exige comprender al sujeto desde su complejidad, dejando de lado visiones reduccionistas que invisibilizan su contexto sociocultural, circunstancias vitales y subjetividad $^{(25)}$.

Chile está en una etapa inicial de la pandemia del COVID-19, de ahí que es importante que se consideren las experiencias de otros países que ya han pasado por el punto más alto de contagios. Uno de los aspectos más destacadas por países que han experimentado las consecuencias sociales del COVID-19, es la importancia de recolectar información en relación a aspectos de salud mental de la población en aislamiento social, que permita orientar medidas de apoyo psicosocial y de salud, especialmente de aquellos grupos de mayor riesgo $^{(22)}$.

\section{Limitaciones de este estudio}

Dentro de las limitaciones de este estudio está el que gran parte de las investigaciones disponibles son en población asiática y los resultados podrían no reflejar la situación en poblaciones que difieren geográfica y socioculturalmente. Otra limitación es la falta de estudios que visibilicen la "pandemia social”, que sin duda tendrá consecuencias que se extenderán más allá del término de la emergencia sanitaria.

\section{CONCLUSIÓN}

Investigaciones recientes dan cuenta que la respuesta psicológica a la actual pandemia muestra amplia variabilidad individual, pudiendo desencadenar sentimientos de miedo, ansiedad o tristeza, con distintos grados de severidad. Las respuestas anteriores, si bien son reacciones normales frente a una catástrofe, las personas en condiciones de alta vulnerabilidad social pueden verse particularmente afectadas. 
En el contexto social actual, las enormes disparidades sociales se han hecho más evidentes con el confinamiento, evidenciándose una necesidad urgente de que los gobiernos diseñen, planifiquen y coordinen respuestas multisectoriales mínimas para proteger y mejorar el bienestar psicosocial de las

\section{REFERENCIAS}

1. World Health Organization. Considerations for quarantine of individuals in the context of containment for coronavirus disease (COVID-19): Interim guidance [Internet] World Health Organization; 2020 March [citado 29 abr 2020]. 4 p. Disponible en: from: https://apps.who.int/iris/ handle/10665/331497

2. European Centre for Disease Prevention and Control [Internet] [citado 29 abr 2020]. Disponible en: https://www.ecdc.europa.eu/en/geographicaldistribution-2019-ncov-cases.

3. World Health Organization. Coronavirus disease 2019 (COVID-19). Situation Report - 72. Data as reported by national authorities by 10.00 CET [Internet] [citado 29 abr 2020]; Disponible en: https://www.who.int/docs/default-source/ coronaviruse/situation-reports/20200401-sitrep72-covid-19.pdf?sfvrsn=3dd8971b_2.

4. Organización Panamericana de la Salud. Protección de la Salud Mental en Situaciones de Epidemias 2006 [Internet] [citado 29 abr 2020]. 36 p. Disponible en: https://www.paho.org/ hq/dmdocuments/2009/Pandemia\%20de $\% 20$ influenza $\% 20 y \% 20$ Salud $\% 20$ mental\%20Esp.pdf

5. Jung SJ, Jun JY. Mental Health and Psychological Intervention Amid COVID-19 Outbreak: Perspectives from South Korea. Yonsei Med J [Internet]. 2020 [citado 20 may 2020]; 61(4): 271-2. Disponible en: https://doi.org/10.3349/ ymj.2020.61.4.271

6. World Health Organization. Supporting older people during the COVID-19 pandemic is everyone's business [Internet]. 3 April, 2020 [citado 29 abr 2020]. Disponible en: http://www.euro. who.int/en/health-topics/health-emergencies/ coronavirus-covid-19/news/news/2020/4/ supporting-older-people-during-the-covid-19pandemic-is-everyones-business

7. Qiu J, Shen B, Zhao M, Wang Z, Xie B, Xu Y. A nationwide survey of psychological distress among Chinese people in the COVID-19 epidemic: implications and policy recommendations. Gen Psychiatr [Internet]. 2020 [citado 20 may personas más vulnerables. Estudios poblacionales, que pongan en el centro la persona, y visualicen a partir de un enfoque biopsicosocial las múltiples dimensiones operando en esta crisis humanitaria, son una urgente necesidad.

2020]; 33: e100213. Disponible en:10.1136/ gpsych-2020-100213

8. Wang C, Pan R, Wan X, Tan Y, Xu L, Ho CS, et al. Immediate psychological responses and associated factors during the initial stage of the 2019 coronavirus disease (COVID-19) epidemic among the general population in China. Int J Environ Res Public Health [Internet]. 2020 [citado 20 may 2020]; 17(5): 1729. Disponible en: https:// doi.org/10.3390/ijerph17051729

9. Cao W, Fang Z, Hou G, Han M, Xu X, Dong J, et al. The psychological impact of the COVID-19 epidemic on college students in China. Psychiatry Res [Internet]. 2020 [citado 20 may 2020]; 287: 112934. Disponible en: https://doi.org/10.1016/j. psychres.2020.112934

10. Lee SA. Coronavirus Anxiety Scale: A brief mental health screener for COVID-19 related anxiety. Death Stud [Internet]. 2020 [citado 20 may 2020]; 44(7): 393-401. Disponible en: https:// doi.org/10.1080/07481187.2020.1748481

11. Roy D, Tripathy S, Kar SK, Sharma N, Verma SK, Kaushal V. Study of knowledge, attitude, anxiety \& perceived mental healthcare need in Indian population during COVID-19 pandemic. Asian J Psychiatr [Internet]. 2020 [citado 18 may 2020]; 51: 102083. Disponible en: https://doi. org/10.1016/j.ajp.2020.102083

12. Zhang J, Lu H, Zeng H, Zhang S, Du Q, Jiang T, Du B. The differential psychological distress of populations affected by the COVID-19 pandemic. Brain Behav Immun [Internet]. 2020 [citado 18 may 2020]; S0889-1591(20):30535-3. Disponible en: https://doi.org/10.1016/j.bbi.2020.04.031

13. Lei L, Huang X, Zhang S, Yang J, Yang L, Xu M. Comparison of Prevalence and Associated Factors of Anxiety and Depression Among People Affected by versus People Unaffected by Quarantine During the COVID-19 Epidemic in Southwestern China. Med Sci Monit [Internet]. 2020 [citado 20 may 2020]; 26: e924609. Disponible en: https://www. medscimonit.com/abstract/index/idArt/924609

14. Liu N, Zhanga F, Weia C, Jiaa Y, Shanga Z, Suna L. Prevalence and predictors of PTSS during COVID-19 outbreak in China hardest- 
hit areas: Gender differences matter. Psychiatry Res [Internet]. 2020 [citado 22 may 2020]; 287: 112921. Disponible en: https://doi.org/10.1016/j. psychres.2020.112921

15. Wang Y, Di Y, Ye J, Wei W. Study on the public psychological states and its related factors during the outbreak of coronavirus disease 2019 (COVID-19) in some regions of China. Psychol Health Med [Internet]. 2020 [citado 20 may 2020]; 1-10. Disponible en: https://doi.org/10.1 080/13548506.2020.1746817

16. Zhang Y, Ma ZF. Impact of the COVID-19 pandemic on mental health and quality of life among local residents in Liaoning Province, China: A cross-sectional study. Int J. Environ Res. Public Health [Internet]. 2020 [cited 2020 april 20]; 17(7): 2381. Disponible en: https://doi. org/10.3390/ijerph17072381

17. Yuan S, Liao Z, Huang $H$, Jiang B, Zhang $X$, Wang Y, et al. Comparison of the Indicators of Psychological Stress in the Population of Hubei Province and Non-Endemic Provinces in China During Two Weeks During the Coronavirus Disease 2019 (COVID-19) Outbreak in February 2020. Med Sci Monit [Internet]. 2020 [citado 18 may 2020]; 26: e923767. Disponible en: 10.12659/ MSM.923767

18. Brooks SK, Webster RK, Smith LE, Woodland L, Wessely S, Greenberg N, et al. The psychological impact of quarantine and how to reduce it: rapid review of the evidence. Lancet [Internet]. 2020 [citado 20 abr 2020]; 395(10227): 912-20. Disponible en: https://doi.org/10.1016/ S01406736(20)30460-8

19. Ornell F, Schuch JB, Sordi AO, Kessler FHP. "Pandemic fear" and COVID-19: mental health burden and strategies. Braz J Psychiatry [Internet]. 2020 [citado 20 abr 2020]; 42(3): 232-35. Disponible en: http://dx.doi.org/10.1590/15164446-2020-0008.

20. Ho CS, Chee CY, Ho RC. Mental health strategies to combat the psychological impact of COVID-19 beyond paranoia and panic. Ann Acad Med Singapore [Internet]. 2020 [citado 20 abr 2020]; 49(3): 155-60. Disponible en: https:// doi.org/10.1590/1516-4446-2020-0008

21. Universidad de Chile. Salud Mental en Situación de Pandemia. Documento para Mesa Social Covid-19 [Internet] Santiago de Chile: Universidad de Chile; 2020 [citado 29 may 2020]. Disponible en: https://www.uchile.cl/portal/especiales/ covid19/163020/estrategia-nacional-para-saludmental-propuesta-a-mesa-social-covid-19

22. Holmes E, Connor R, Perry H, Tracey I, Wessely $S$ et.al. Multidisciplinary research priorities for the
COVID-19 pandemic: a call for action for mental health science. Lancet Psychiatry [Internet]. 2020 [citado 20 may 2020]; S2215-0366(20): 30168-1. Disponible en: https://doi.org/10.1016/S22150366(20)30168-1

23. Kessler G, Bermúdez N, Binstock G, Cerrutti M, Pecheny M, Piovani JI, et al. Relevamiento del impacto social de las medidas del Aislamiento dispuestas por el PEN. Comisión de Ciencias Sociales de la Unidad Coronavirus COVID-19 [Internet] Buenos Aires, Argentina: MINCYTCONICET-AGENCIA; 2020 [citado 20 abr 2020]. Disponible en: https://www.conicet.gov. ar/wp-content/uploads/Informe_Final_Covid-Cs. Sociales-1.pdf

24. Bäuerle A, Skoda EM, Dörrie N, Böttcher J, Teufel M. Psychological support in times of COVID-19: the Essen community-based CoPE concept. J Public Health (Oxf) [Internet]. 2020 [citado 20 abr 2020]; pii: fdaa053. Disponible en: 10.1093/ pubmed/fdaa053. [Epub ahead of print] PMID: 32307516

25. Zarebski G. La identidad flexible como Factor Protector en el curso de la Vida [Internet] Buenos Aires, Argentina: Universidad de Maimonides. UMAI. 2019 [citado 20 abr 2020]. Disponible en: https://gerontologia.maimonides.edu/2019/04/ la-identidad-flexible-como-factor-protector-en-elcurso-de-la-vida/

26. Duan L, Zhu G. Psychological interventions for people affected by the COVID-19 epidemic. Lancet Psychiatry [Internet]. 2020 [citado 29 abr 2020]; 7(4): 300-2. Disponible en: https://doi. org/10.1016/S2215-0366(20)30073-0

27. Cowan K. Survey results: Understanding people's concerns about the mental health impacts of the COVID-19 pandemic. The Academy of Medical Sciences [Internet]. 2020 [citado 20 may 2020]; 36 p. Disponible en: http://www.acmedsci.ac.uk/ COVIDmentalhealthsurveys

28. Valtorta NK, Kanaan M, Gilbody S, Hanratty B. Loneliness, social isolation and risk of cardiovascular disease in the English Longitudinal Study of Ageing. Eur J Prev Cardiol [Internet]. 2018 [citado 20 abr 2020]; 25(13): 1387-96. Disponible en: doi.org/10.1177/2047487318792696

29. Gunnell D, Appleby L, Arensman E, Hawton $\mathrm{K}$, John A, Kapur N, et al. Suicide risk and prevention during the COVID-19 pandemic. Lancet Psychiatry [Internet]. 2020 [citado $20 \mathrm{abr}$ 2020]; S2215-0366(20): 30171-1. Disponible en: 10.1016/S2215-0366(20)30171-1.

30. Yip PS, Cheung YT, Chau PH, Law YW. The impact of epidemic outbreak: The case of severe acute respiratory syndrome (SARS) and suicide among 
older adults in Hong Kong. Crisis [Internet]. 2020 [citado 20 abr 2020]; 31(2): 86-92. Disponible en: https://doi.org/10.1027/0227-5910/a000015

31. Tsang HW, Scudds RJ, Chan EY. Psychosocial impact of SARS. Emerg Infect Dis [Internet]. 2004 [citado abr 2020]; 10(7): 1326-27. Disponible en: https://stacks.cdc.gov/view/cdc/14600

32. Nickell LA, Crighton, EJ, Tracy CS, Al-Enazy H, Bolaji Y, Hanjrah S, et al. Psychosocial effects of SARS on hospital staff: survey of a large tertiary care institution. CMAJ [Internet]. 2004 [citado $20 \mathrm{abr}$ 2020]; 170(5): 793-98. Disponible en: https://doi. org/10.1503/cmaj.1031077

33. Frasquilho D, Matos M, Salonna F, Guerreiro D, Storti CC, Gaspar T, et al. Mental health outcomes in times of economic recession: a systematic literature review. BMC Public Health [Internet]. 2016 [citado 20 abr 2020]; 16: 115. Disponible en: https://doi.org/10.1186/s12889-016-2720-y

34. Berriel F, Carbajal M, Castro G, Guidotti C, Lladó M, Martínez F, et al. Aportes del Núcleo Interdisciplinario de Estudios sobre la Vejez y el Envejecimiento (NIEVE) para el trabajo en Salud Mental con Personas Mayores en el marco de la situación sanitaria por COVID 19, desde una perspectiva de DDHH [Internet]. Montevideo, Uruguay: NIEVE, Universidad de la República; 2020 [citado 17 abr 2020]. Disponible en: https://www.gerontologia.org/portal/information/ showInformation.php?idinfo $=4503$ 